\title{
Effect of Lipodox in combination with bevacizumab in a patient with a metastatic malignant phyllodes breast tumor: A case report
}

\author{
CHIN CHENG SU ${ }^{1-4}$, CHIH-JUNG $\mathrm{CHEN}^{5-7}$ and SHOU-JEN KUO ${ }^{2,3}$ \\ ${ }^{1}$ Tumor Research Center of Integrative Medicine; ${ }^{2}$ Comprehensive Breast Cancer Center; ${ }^{3}$ Department of Surgery, \\ Changhua Christian Hospital, Changhua 50006; ${ }^{4}$ School of Chinese Medicine, College of Chinese Medicine, \\ China Medical University, Taichung 40402; ${ }^{5}$ Department of Surgical Pathology, Changhua Christian Hospital, \\ Changhua 50006; ${ }^{6}$ School of Medicine, Chung Shan Medical University, Taichuang 40402; ${ }^{7}$ Department of Medical \\ Technology, Jen-Teh Junior College of Medicine, Nursing and Management, Miaoli 360, Taiwan, R.O.C.
}

Received July 22, 2016; Accepted July 20, 2017

DOI: $10.3892 / 01.2017 .7094$

\begin{abstract}
A 76-year-old female patient with a malignant phyllodes tumor underwent modified radical mastectomy and wide excision. Multiple nodules were observed in the operated wound area. Positron emission tomography-computed tomography (PET-CT) revealed recurrent disease in the left breast, the adjacent left third rib, the left internal mammary region and the left ilium. A novel formulation of bevacizumab $\left(5 \mathrm{mg} / \mathrm{m}^{2}\right.$, first day) in combination with liposomal doxorubicin (Lipodox, $30 \mathrm{mg} / \mathrm{m}^{2}$, second day) was administered for 3 cycles every 2 weeks, and subsequently wide excision was performed. Lipodox $\left(40 \mathrm{mg} / \mathrm{m}^{2}\right)$ was administered for 3 cycles every 3 weeks, starting 4 weeks after the surgery. Follow-up whole body PET-CT scanning, 3 and 6 months later, indicated no sign of residual hypermetabolic malignancy. Malignant phyllodes tumors do not usually respond to chemotherapy or radiotherapy. In the present case report, a novel formulation of bevacizumab in combination with Lipodox was administered as neoadjuvant chemotherapy in a patient with a malignant phyllodes tumor and preoperative tumor shrinkage was achieved, resulting in clear resection margins.
\end{abstract}

\section{Introduction}

The phyllodes tumors are rare breast tumors that account for $<1 \%$ of breast neoplasms worldwide (1). They are fibroepithelial tumors resembling fibroadenomas but have a predominant connective tissue component. They are divided into three categories: Benign, borderline and malignant (2). Wide excision with clear margins is typically performed and prognosis

Correspondence to: Dr Chin Cheng Su, Tumor Research Center of Integrative Medicine, Changhua Christian Hospital, 135 Nan-Hsiao Street, Changhua 50006, Taiwan, R.O.C.

E-mail: succ.maeva@msa.hinet.net

Key words: Lipodox, bevacizumab, malignant phyllodes tumor depends on the histological characteristics of the connective tissue component (3). Multivariate analysis indicated benign histology, negative tumor margins and absence of residual disease following initial treatment and radiation therapy as favorable independent prognostic factors (4). However, the role of neoadjuvant treatment in phyllodes tumors remains unclear and requires elucidation on a case-by-case basis (5).

\section{Case report}

A 77-year-old female patient from Changhua City underwent simple mastectomy on 25 February 2015 in Changhua Christian Hospital (Changhua, Taiwan) for a malignant phyllodes tumor in the left breast. Pathological examination revealed a soft tumor mass, $13 \times 12 \mathrm{~cm}$ in size, extended in the whole breast. A rapidly progressing $>5 \mathrm{~cm}$ mass, with bleeding and a central necrosis area, was observed on 25 April 2015. A recurrent phyllodes tumor was suspected and the patient underwent a second surgery on 28 April 2015. Pathological examination revealed a protruding tumor, $10 \times 4.5 \times 4.8 \mathrm{~cm}$ in size, associated with skin ulceration. Multiple nodules were observed in the operated wound area 10 days after the second surgery and the patient was admitted to the Breast Surgery Outpatient Department (Changhua Christian Hospital). During initial physical examination, the patient was afebrile with normal vital signs. Examination of the left breast revealed multiple lobulated masses with irritated overlying skin. The right breast was normal. Positron emission tomography-computed tomography (PET-CT) and magnetic resonance imaging (MRI) were performed for further evaluation. $\mathrm{CT}$ and $\left[{ }^{18} \mathrm{~F}\right]$ fluorodeoxyglucose (FDG)-PET scans revealed multiple areas with markedly increased FDG uptake in the left breast, the adjacent left third rib and the left internal mammary region. These areas demonstrated increased standardized uptake values in the subsequent analysis, suggesting malignancy (Fig. 1). MRI examination revealed an ovoid heterogeneous enhancement mass $5.0 \times 4.3 \times 3.3 \mathrm{~cm}$ in the left breast (lower-outer quadrant), along with a $3.7 \times 3.2 \times 2.3 \mathrm{~cm}$ mass within the pectoralis muscle layer (Fig. 2).

Malignant phyllodes tumors are rare and aggressive fibroepithelial neoplasms. An accurate diagnosis of metastasis should 
be based on clinicopathological examinations. To achieve successful management of these tumors, early detection and complete resection prior to dissemination are of marked importance (6). The present case report describes a 46-year-old female patient with a metastatic phyllodes tumor in the anterior chest wall. Even though it was not possible to treat the lesion by surgery or chemotherapy, the tumor size was markedly decreased following radiation therapy, one of the palliative therapies (7). The present case report suggests that radiation therapy should be recognized as a treatment of choice for palliative medicine. Hashimoto et al (8) reported a case of preoperative chemoembolization of a large malignant phyllodes tumor in which marked tumor shrinkage was achieved prior to surgery. Intra-arterial Epirubicin infusion and subsequent embolization with Embosphere microspheres were administered three times over the course of 6 weeks. The patient underwent surgery without skin grafting, and no local recurrence or distant metastasis was observed within 6 months of surgery.

Pathological examination was performed in samples obtained from the first and second surgery. Samples were fixed in $10 \%$ neutralized formalin at room temperature for $24 \mathrm{~h}$. Paraffin embedded tumor tissue sections $(4 \mu \mathrm{m})$ were placed on coated slides and washed with xylene to remove the paraffin, then rehydrated using serial dilutions of alcohol (70 and 90\%, respectively). Sections were washed with $\mathrm{PBS}$ ( $\mathrm{pH}$ 7.2) and incubated with anti-cluster of differentiation 34 (CD34) antibody (1:50 dilution; Thermo Fisher Scientific, Inc., Waltham, MA, USA; clone QBEend/10; ab81289) for $60 \mathrm{~min}$ at room temperature. Subsequently, slides were washed 3 times with PBS and the conventional streptavidin peroxidase method (LSAB kit K675; Dako; Agilent Technologies, Inc., Santa Clara, CA, USA) was performed for signal development. Incubation with ready-to-use secondary antibodies from the kit was performed at room temperature for 10-30 mins. PBS, instead of primary antibodies, was used as a negative control. Stromal endothelial cells were used as a positive control. Samples were viewed under a light microscope (magnification, $\mathrm{x} 40$ ).

Paraffin-embedded tumor tissue sections $(4 \mu \mathrm{m})$ were processed with standard hematoxylin and eosin staining using Tissue-Tek DRS (Sakura Finetek Europe B.V., Flemingweg, The Netherlands), according to the manufacturer's protocol. Hypercellular areas with malignant tumor cells (Fig. 3A) and abundant intratumoral vessels (Fig. 3B), immunoreactive for the hematopoietic progenitor cell antigen CD34 were observed in the primary tumor prior to chemotherapy. Similarly, hypercellular areas with atypical tumor cells (Fig. 3C) and abundant intratumoral vessels immunoreactive for CD34 (Fig. 3D) were observed in the recurrent malignant phyllodes tumor prior to chemotherapy. Owing to the patient's advanced age and the multiple nodules, bevacizumab $\left(5 \mathrm{mg} / \mathrm{m}^{2}\right)$ on the first day in combination with Lipodox $\left(30 \mathrm{mg} / \mathrm{m}^{2}\right)$ on the second day was administered for 3 cycles every 2 weeks, commencing on 22 May 2015. The patient's clinical condition improved significantly. A PET-CT follow-up scan was performed on 13 July 2015 and revealed a postoperative alteration in the left breast and a small metastatic lymph node in the left axillary region (Fig. 4). A recurrent left breast phyllodes tumor was suspected and wide surgical excision was conducted on 27 July 2015 (Fig. 5A). The sample obtained was $16 \times 11 \times 3 \mathrm{~cm}$ in size and consisted of breast tissue, pectoral fascia and muscle fibers in fresh state (Fig. 5B).

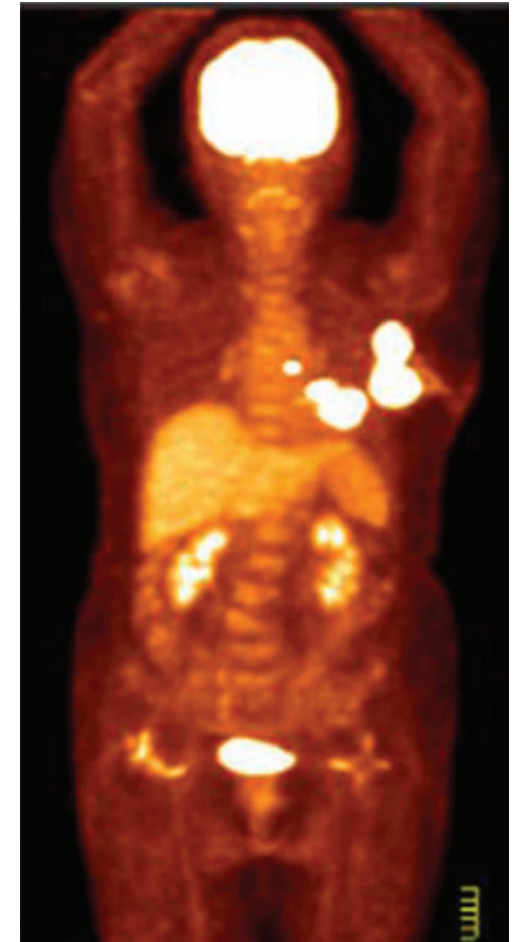

Figure 1. FDG-positron emission tomography-computed tomography was performed on 14 May 2015. Multiple areas with markedly increased FDG uptake were observed in the left breast, the adjacent left third rib and the left internal mammary region. Increased standardized uptake values in the subsequent analysis suggested malignancy. FDG, $\left[{ }^{18} \mathrm{~F}\right]$ fluorodeoxyglucose.

The breast sample was covered by skin with dimensions $7 \times 5.5 \mathrm{~cm}$. Surgical scars were observed on the skin. However, there was no evidence of edema or inflammation. The areola and nipple were not observed. Following tissue sectioning, two nodules measuring $4.2 \times 2.5 \times 2.3 \mathrm{~cm}$ and $2.2 \times 2 \times 1.8 \mathrm{~cm}$ in size, respectively, were revealed. The tumors presented yellowish with necrotic features and the distance between them was $0.5 \mathrm{~cm}$. They were located 0.3 and $1.5 \mathrm{~cm}$ underneath the skin, respectively, and $<0.1 \mathrm{~cm}$ above the pectoral fascia and muscle. The lower peripheral margin measured $0.2 \mathrm{~cm}$. No clear evidence of invasion in the overlying skin was observed. The parenchyma in the remaining breast was not marked. Microscopically, sections of the breast exhibited extensive necrotic areas and hemorrhagic regions surrounded by granulation tissue with chronic inflammation, hyalinization and foreign body reaction with multinucleated giant cells. Hemosiderin deposition was also observed. The presentation was consistent with post-treatment appearance. Following chemotherapy, the tumor exhibited marked necrosis. Scant and residual tumor cells (Fig. 6A) along with decreased intratumoral vessels, as demonstrated by CD34 immunostaining, were observed (Fig. 6B). Lipodox $\left(40 \mathrm{mg} / \mathrm{m}^{2}\right)$ was administered for 3 cycles every 3 weeks and surgical wide excision was conducted 4 weeks later. PET-CT was performed on 16 October 2015. Postoperative alterations along with post-treatment reaction were observed in the left lateral chest wall. No evident metastatic lesion was observed (Fig. 7). A follow-up PET-CT scan was performed on 15 January 2016 and no evidence of residual hypermetabolic malignancy was detected. Compared with the previous scan (on 16 October 2015), persistent complete metabolic response was indicated 

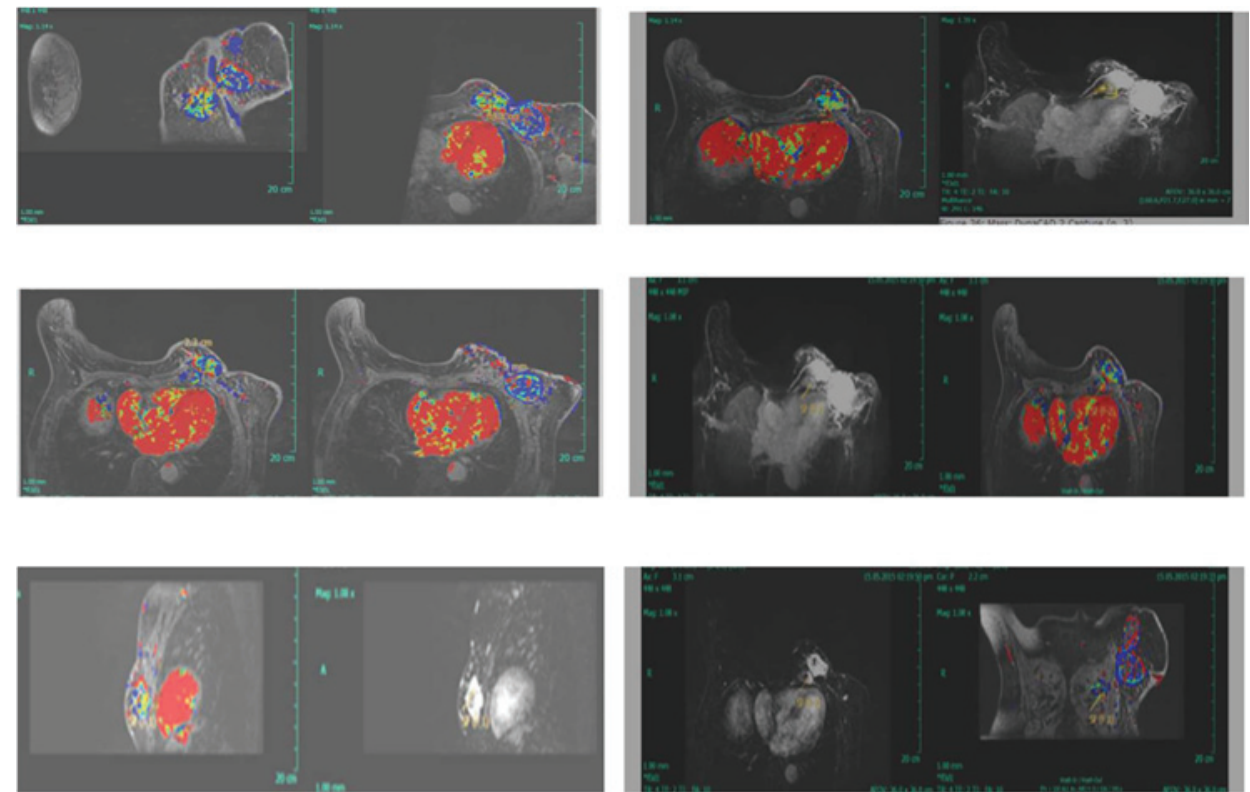

Figure 2. Breast magnetic resonance imaging was performed on 15 May 2015. An ovoid heterogeneous enhancement mass $5.0 \times 4.3 \times 3.3 \mathrm{~cm}$ was revealed in the left breast area (lower-outer quadrant) along with a mass $3.7 \times 3.2 \times 2.3 \mathrm{~cm}$ within the pectoralis muscle layer.

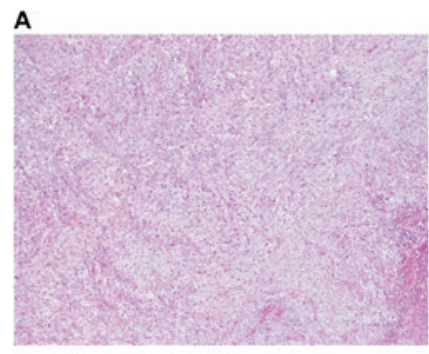

C

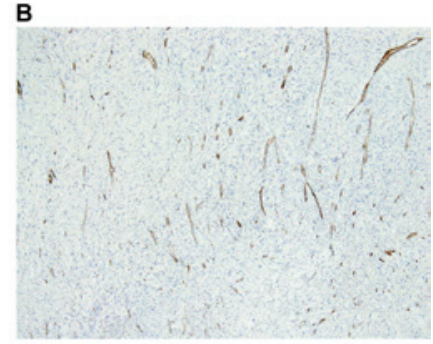

D

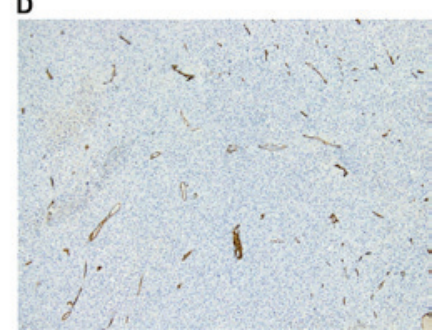

Figure 3. Representative microscopic images of the malignant phyllodes tumor prior to chemotherapy. (A) Hypercellular areas with tumor cells and (B) abundant intratumoral vessels, immunoreactive for CD34, were observed in the primary malignant phyllodes tumor tissue. (C) Hypercellular areas with atypical tumor cells and (D) abundant intratumoral vessels immunoreactive for CD34 were observed in the recurrent malignant phyllodes tumor tissue. Magnification, x40. CD, cluster of differentiation.

(Fig. 8). Written informed consent was obtained from the patient prior to publication of the present study.

\section{Discussion}

Malignant breast phyllodes tumors frequently recur following breast-conserving surgery (9). The high rate of local recurrence renders the research for therapeutic improvement highly important. It is well-documented that adjuvant radiation therapy decreases the local recurrence

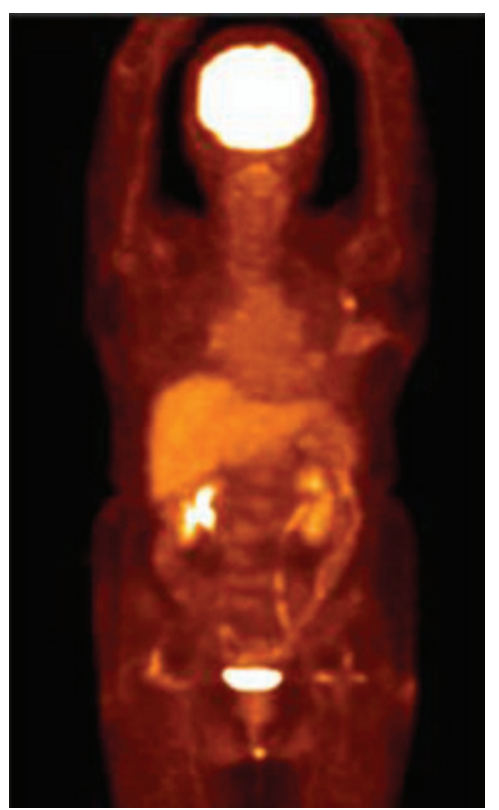

Figure 4. Positron emission tomography-computed tomography follow-up scan performed on 13 July 2015. A postoperative alteration in the left breast and a small metastatic lymph node in the left axillary region were observed.

rate of borderline and malignant phyllodes tumors in patients undergoing breast conserving surgery. However, to the best of our knowledge, an effect on overall or disease-free survival has not been reported $(10,11)$. Surgical wide excision with a clear margin is considered the treatment of choice. Patients with tumors with infiltrating margins, stromal overgrowth and hypercellularity are at a high risk of metastasis (12). Several predictive factors of recurrence and metastasis, including positive surgical margins, increased mitotic activity, stromal atypia, stromal hypercellularity and overgrowth have been described. However, the role 

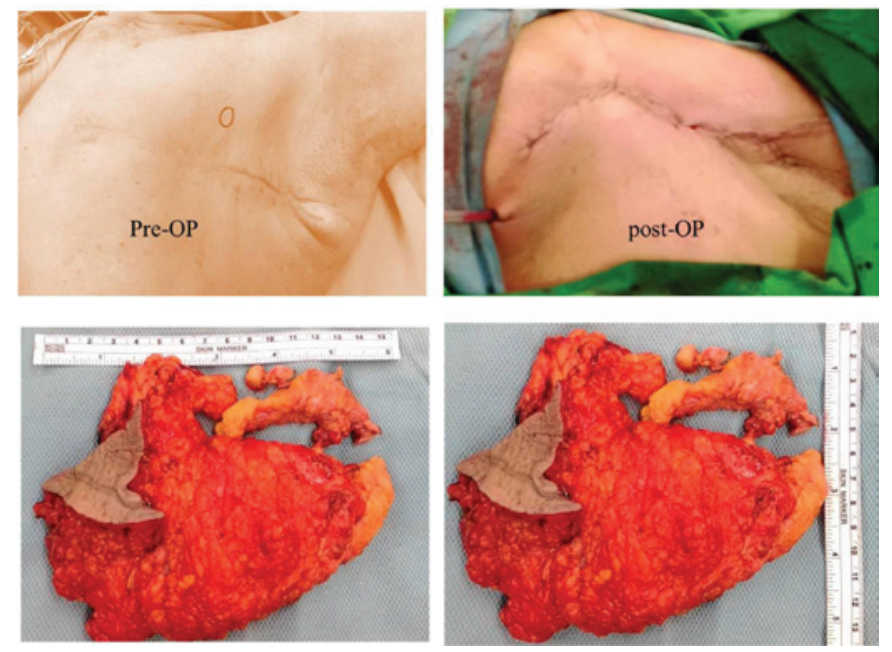

Figure 5. Wide surgical excision was conducted on 27 July 2015. The sample obtained was $16 \times 11 \times 3 \mathrm{~cm}$ in size and consisted of breast tissue, pectoral fascia and muscle fibers in fresh state. OP, surgery.

A

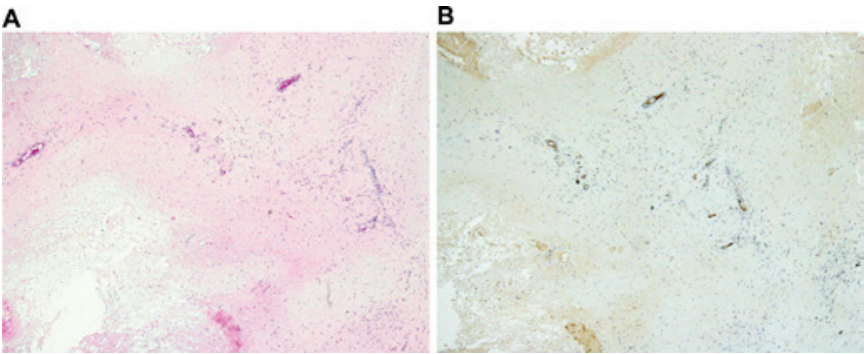

Figure 6. Representative microscopic images of the malignant phyllodes tumor following chemotherapeutic treatment. (A) Marked tumor necrosis with scant and residual tumor cells and (B) decreased intratumoral vessels, as demonstrated by cluster of differentiation 34 immunostaining, were observed. Magnification, x40.

of adjuvant therapies (radiotherapy and chemotherapy) remains unclear (13). Lipodox is used as a monotherapy for the treatment of metastatic breast cancer, where there is increased cardiac risk (www.drugs.com/pro/lipodox.html) . Several agents targeting the vascular endothelial growth factor pathway have been used in combinatorial strategies and improved the efficacy of other anticancer drugs for the treatment of lung, stomach, colorectal, ovarian and breast carcinomas (14). A meta-analysis study of randomized controlled trials demonstrated that in the neoadjuvant setting, bevacizumab in combination with chemotherapy compared with chemotherapy alone increased the percentage of patients with non-metastatic breast cancer that achieved a pathological complete response. Bevacizumab was particularly effective in patients with human epidermal growth factor receptor 2-negative and hormone receptor-negative breast tumors (15). Malignant phyllodes tumors do not respond to chemotherapy or radiotherapy. The novel formulation of bevacizumab $\left(5 \mathrm{mg} / \mathrm{m}^{2}\right.$, first day) in combination with Lipodox (30 mg/m², second day), twice a week, achieved sufficient tumor shrinkage as neoadjuvant chemotherapy. To the best of our knowledge, effective neoadjuvant treatment of malignant phyllodes tumors resulting in clear margins following resection has not been reported previously.

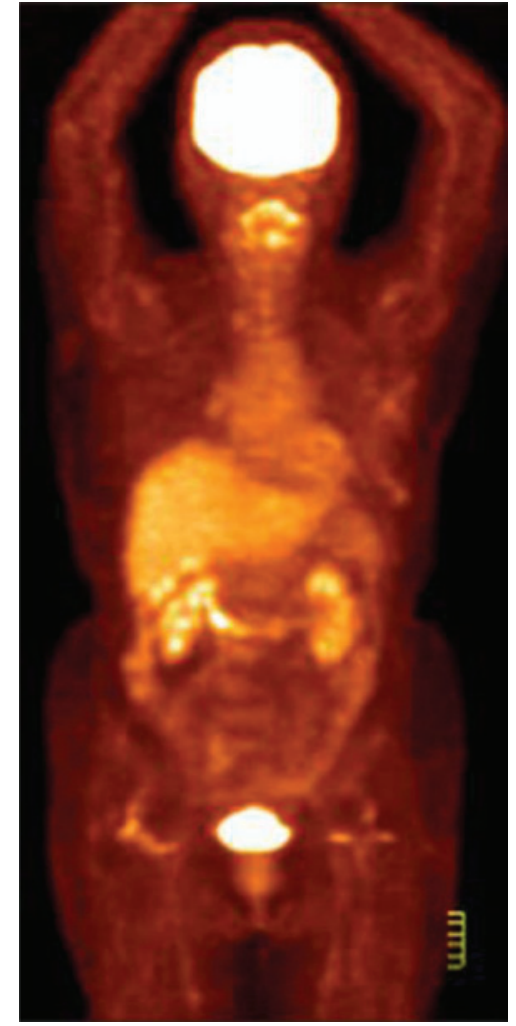

Figure 7. Positron emission tomography-computed tomography performed on 13 October 2015. Postoperative alterations along with post-treatment reaction were observed in the left lateral chest wall. No evident metastatic lesion was observed.

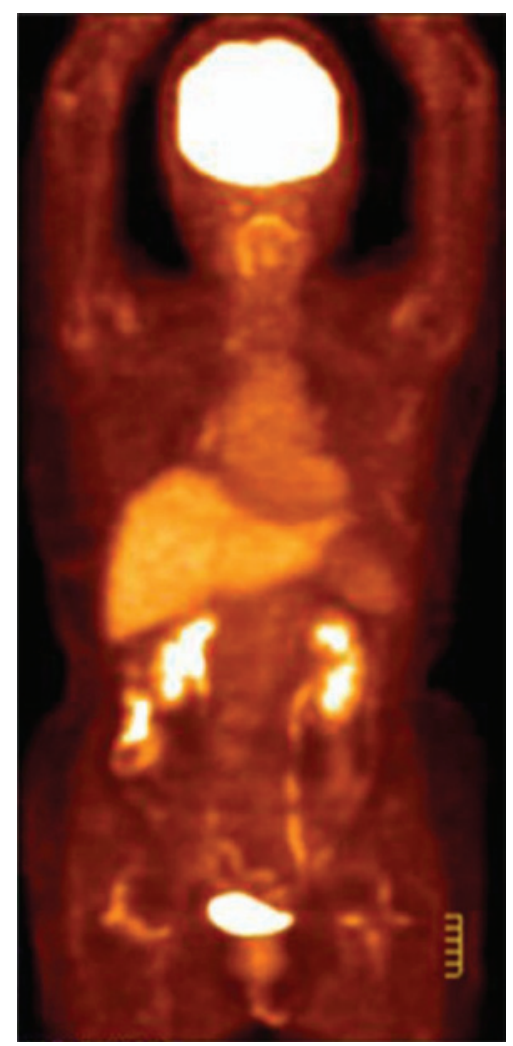

Figure 8. Follow-up PET-CT performed on 15 January 2016. No evidence of residual hypermetabolic malignancy was observed. Compared with the previous PET-CT scan (on 16 October 2015) persistent complete metabolic response was considered. PET-CT, positron emission tomography-computed tomography. 


\section{References}

1. Nathan Roberts* and Dianne M. Runk: Aggressive malignant phyllodes tumor. Int J Surg Case Rep 8: 161-165, 2015.

2. Norat F, Dreant N, Riah Y and Lebreton E: Extraordinary case of malignant phylloid tumor of the breast: Surgical reconstruction treatment. Ann Ital Chir 80: 475-478, 2009 (In Italian).

3. Matar N, Soumani A, Noun M, Chraibi T, Himmi A, el Mansouri A, Aderdour M and Bekkay M: Phyllodes tumors of the breast. Forty one cases. J Gynecol Obstet Biol Reprod (Paris) 26: 32-36, 1997.

4. Belkacémi Y, Bousquet G, Marsiglia H, Ray-Coquard I, Magné N, Malard Y, Lacroix M, Gutierrez C, Senkus E, Christie D, et al: Phyllodes tumor of the breast. Int J Radiat Oncol Biol Phys 70 492-500, 2008.

5. Guillot E, Couturaud B, Reyal F, Curnier A, Ravinet J, Laé M, Bollet M, Pierga JY, Salmon R and Fitoussi A; Breast Cancer Study Group of the Institut Curie: Management of phyllodes breast tumors. Breast J 17: 129-137, 2011.

6. EI Ochi MR, Toreis M, Benchekroun M, Benkerroum Z, Allaoui M, Ichou M, El Khannoussi B, Albouzidi A and Oukabli M: Bone metastasis from malignant phyllodes breast tumor: Report of two cases. BMC Clin Pathol 16: 4 , 2016.

7. Sazuka T, Matsuzaki H, Kanada Y, Tohnosu N, Yoshiwara C, Aruga T, Iwata K, Sasahara N, Kobayashi H, Yokoyama M, et al: A case of malignant phyllodes tumor effectively treated by radiation therapy as a palliative medicine. Gan To Kagaku Ryoho 42: 1698-1699, 2015 (In Japanese).
8. Hashimoto K, Mimura H, Arai Y, Doi M, Kojima Y, Tsugawa K and Nakajima Y: Successful preoperative chemoembolization in the treatment of a giant malignant phyllodes tumor. Cardiovasc Intervent Radiol 39: 1070-1075, 2016.

9. Barth RJ Jr: Histologic features predict local recurrence after breast conserving therapy of phyllodes tumors. Breast Cancer Res Treat 57: 291-295, 1999.

10. Zeng S, Zhang X, Yang D, Wang X and Ren G: Effects of adjuvant radiotherapy on borderline and malignant phyllodes tumors: A systematic review and meta-analysis. Mol Clin Oncol 3: 663-671, 2015.

11. Gnerlich JL, Williams RT, Yao K, Jaskowiak N and Kulkarni SA: Utilization of radiotherapy for malignant phyllodes tumors: Analysis of the National Cancer Data Base, 1998-2009. Ann Surg Oncol 21: 1222-1230, 2014.

12. Chen WH, Cheng SP, Tzen CY, Yang TL, Jeng KS, Liu CL and Liu TP: Surgical treatment of phyllodes tumors of the breast: Retrospective review of 172 cases. J Surg Oncol 91: 185-194, 2005.

13. Khosravi-Shahi P: Management of non metastatic phyllodes tumors of the breast: Review of the literature. Surg Oncol 20: e143-e148, 2011.

14. Arjaans M, Schröder CP, Oosting SF, Dafni U, Kleibeuker JE and de Vries EG: VEGF pathway targeting agents, vessel normalization and tumor drug uptake: From bench to bedside. Oncotarget 7: 21247-21258, 2016.

15. Cao L, Yao GY, Liu MF, Chen LJ, Hu XL and Ye CS: Neoadjuvant bevacizumab plus chemotherapy versus chemotherapy alone to treat non-metastatic breast cancer: A meta-analysis of randomised controlled trials. PLoS One 10: e0145442, 2015. 